\title{
Era of Personal Health Records in Korea
}

\author{
Jae-Ho Lee ${ }^{1,2}$ \\ 'Department of Emergency Medicine, Asan Medical Center, University of Ulsan College of Medicine, Seoul, Korea \\ ${ }^{2}$ Department of Information Medicine, Asan Medical Center, University of Ulsan College of Medicine, Seoul, Korea
}

In line with the global trend of digital transformation, the Korean Ministry of Health and Welfare is strongly promoting strategies for the digital transformation of healthcare. Promising digital health technologies, such as mobile health, digital therapeutics, social robots, and virtual reality, are patient-centered technologies. These technologies should not only help to care for patients, but should also be constructed to reflect patients' needs and values. For these purposes, patients' medical information and health data must be integrated and linked with these technologies. Personal health records (PHRs) are the technology that makes this possible. Digital transformations in healthcare that are valuable to patients are possible only if patient information flows safely without blockage. PHRs are known to provide various benefits to healthcare stakeholders, including patients [1]. Significant results have been reported not only in terms of clinical outcomes, but also for patient activation, engagement, satisfaction, and safety.

Developed countries provide PHRs that allow patients to access their medical information and manage their health data. The United States enacted the Health Information Technology for Economic and Clinical Health (HITECH) Act in 2009 and invested a substantial amount of budget and resources to ensure that certified Electronic Medical Records (EMRs) were established in clinics and hospitals [2]. The certified EMR requirements include a patient portal that provides medical information to patients as an essential

This is an Open Access article distributed under the terms of the Creative Commons Attribution Non-Commercial License (http://creativecommons.org/licenses/by$\mathrm{nc} / 4.0 /$ ) which permits unrestricted non-commercial use, distribution, and reproduction in any medium, provided the original work is properly cited.

(c) 2022 The Korean Society of Medical Informatics function. In Meaningful Use (MU) stage 2, patients can use the patient portal to access, download, and transmit their information. The PHR adoption rate sharply increased after MU stage 2. The 2021 Health Information National Trend Survey found that $39.5 \%$ of respondents had accessed their medical information online at least once in the past year [3]. This policy was incorporated into several provisions in the 21st Century Cures Act, which was passed in 2016 and came into force in 2021 [2].

National-level PHR services are provided as patient-accessible Electronic Health Records in Europe and Australia. According to a 2017 OECD report, more than half of the 38 countries provided PHR services to patients [4]. England launched a mobile PHR service called the NHS App on December 31,2018. This app was downloaded more than YouTube, TikTok, and Instagram in England in 2021, as the coronavirus disease 2019 (COVID-19) official vaccination passport function was included [5]. Furthermore, in Finland's My Kanta and Australia's My Health Record (MyHR) services, access to vaccination information has skyrocketed since the COVID-19 pandemic. My Kanta is used by more than $51 \%$ of the population in Finland, and Journalen in Sweden is used by more than $30 \%$ of the population $[6,7]$.

Korea has a very high EMR adoption rate, but PHR service is not active. In 2021, the government promoted the My Healthway project and started a mobile-based public PHR app service. Although the information and functions provided by this app have many limitations, users' satisfaction is high. This app provides users with integrated medication and vaccination information, and provides services linked to Samsung Health and Apple HealthKit. Services that interoperate with hospitals' EMRs are being promoted.

The Healthcare Informatization Status Survey in 2020 re- 
vealed that mobile PHRs were provided by $2.4 \%$ of general hospitals and $62 \%$ of tertiary general hospitals [8]. Hospitals preferred mobile access over a web platform. However, very few hospitals provide the core functions of PHRs. According to the survey findings, only $64.3 \%$ of tertiary general hospitals and $3.3 \%$ of general hospitals provided patients with access to their medical information. The opportunity for patients to download their medical information was very low, as $9.5 \%$ of tertiary general hospitals provided a function to download medication information, $7.1 \%$ provided a function to download test results, and $4.8 \%$ enabled downloads of a health summary. No hospitals allowed patients to download vaccination information, allergy information, or vital signs. The patient-generated health data entry function was also rare. Above all, there were only few hospitals that planned to provide these functions within 3 years.

Private hospitals provide PHRs voluntarily without institutional support. Because they do not have sufficient financial resources and an adequate business model to maintain this service, the opportunity to expand and enhance PHR functions is very low. The Korean government is actively promoting a policy of linking EMR and PHR information of private hospitals on the My Healthway platform, but there is not a meaningful policy to support hospitals' PHRs. Since the private sector accounts for more than $80 \%$ of healthcare services in Korea, it is necessary to support the provision of high-quality PHR services by private hospitals and to establish a system that interoperates with public PHR services. The use of PHRs by patients and clinicians should be accompanied by major changes in the healthcare system and related fields. Sufficient resources and a long-term plan should be prepared to reduce Korea's institutional and technological gap with other developed centuries and to grow digital health technology and industry.

Health is the most important value for citizens in modern society, and healthcare is the field that can benefit the most from digital transformation. It is necessary to concentrate on enhancing the capabilities of healthcare stakeholders to introduce and spread PHR services in Korea.

\section{Conflict of Interest}

No potential conflict of interest relevant to this article was reported.

\section{ORCID}

Jae-Ho Lee (http://orcid.org/0000-0003-2619-1231)

\section{References}

1. Neves AL, Freise L, Laranjo L, Carter AW, Darzi A, Mayer E. Impact of providing patients access to electronic health records on quality and safety of care: a systematic review and meta-analysis. BMJ Qual Saf 2020; 29(12):1019-32.

2. Everson J, Patel V, Adler-Milstein J. Information blocking remains prevalent at the start of 21st Century Cures Act: results from a survey of health information exchange organizations. J Am Med Inform Assoc 2021; 28(4):727-32.

3. Health Information National Trend Survey. Trends and disparities in patient portal use (HINTS Briefs 45) [Internet]. Bethesda (MD): National Cancer Institute; 2021 [cited at 2022 Feb 3]. Available from: https://hints.cancer.gov/docs/Briefs/HINTS_Brief_45.pdf.

4. Oderkirk J. Readiness of electronic health record systems to contribute to national health information and research. Paris, France: Organisation for Economic Cooperation and Development; 2017.

5. Hodes S, Majeed A. Using the NHS App as a covid-19 vaccine passport. BMJ 2021;373:n1178.

6. Jormanainen V, Parhiala K, Niemi A, Erhola M, Keskimaki I, Kaila M. Half of the Finnish population accessed their own data: comprehensive access to personal health information online is a corner-stone of digital revolution in Finnish health and social care. Finn J eHealth eWelf 2019;11(4):298-310.

7. Hagglund M, DesRoches C, Petersen C, Scandurra I. Patients' access to health records. BMJ 2019;367:15725.

8. Korea Health Information Service. 2020 Healthcare Informatization Status Survey Report [Internet]. Seoul, Korea: Korea Health Information Service; 2021 [cited at $2022 \mathrm{Feb} 3$ ]. Available from: https://www.k-his.or.kr/ board.es? $\mathrm{mid}=\mathrm{a} 10306040000 \& \mathrm{bid}=0005 \& \mathrm{tag}=\& \mathrm{act}=\mathrm{vie}$ w\&list_no $=283$. 